\title{
Longitudinal assessment of IFN-I activity and immune profile in critically ill COVID-19 patients with acute respiratory distress syndrome
}

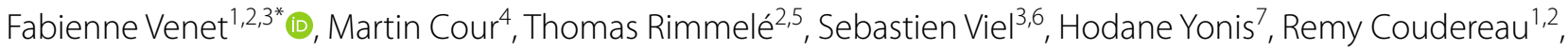
Camille Amaz ${ }^{8}$, Paul Abraham ${ }^{5}$, Céline Monard ${ }^{5}$, Jean-Sebastien Casalegno ${ }^{9}$, Karen Brengel-Pesce ${ }^{2}$, Anne-Claire Lukaszewicz ${ }^{2,5}$, Laurent Argaud ${ }^{4}$ and Guillaume Monneret ${ }^{1,2}$ on behalf of the RICO study group

\begin{abstract}
Background: Since the onset of the pandemic, only few studies focused on longitudinal immune monitoring in critically ill COVID-19 patients with acute respiratory distress syndrome (ARDS) whereas their hospital stay may last for several weeks. Consequently, the question of whether immune parameters may drive or associate with delayed unfavorable outcome in these critically ill patients remains unsolved.

Methods: We present a dynamic description of immuno-inflammatory derangements in 64 critically ill COVID-19 patients including plasma IFNa2 levels and IFN-stimulated genes (ISG) score measurements.

Results: ARDS patients presented with persistently decreased lymphocyte count and mHLA-DR expression and increased cytokine levels. Type-I IFN response was initially induced with elevation of IFNa2 levels and ISG score followed by a rapid decrease over time. Survivors and non-survivors presented with apparent common immune responses over the first 3 weeks after ICU admission mixing gradual return to normal values of cellular markers and progressive decrease of cytokines levels including IFNa2. Only plasma TNF-a presented with a slow increase over time and higher values in non-survivors compared with survivors. This paralleled with an extremely high occurrence of secondary infections in COVID-19 patients with ARDS.
\end{abstract}

Conclusions: Occurrence of ARDS in response to SARS-CoV2 infection appears to be strongly associated with the intensity of immune alterations upon ICU admission of COVID-19 patients. In these critically ill patients, immune profile presents with similarities with the delayed step of immunosuppression described in bacterial sepsis.

Keywords: COVID-19, ARDS, Type-I IFN, Immune profile, Immunosuppression

*Correspondence: fabienne.venet@chu-lyon.fr 1 Immunology Laboratory, Hôpital E. Herriot - Hospices Civils de Lyon, 5 place d'Arsonval, 69437 Lyon Cedex 03, France

Full list of author information is available at the end of the article

\section{Introduction}

The severe acute respiratory coronavirus-2 (SARS$\mathrm{CoV}-2)$ is responsible for coronavirus disease-19 (COVID-19) that mostly associates with asymptomatic and mild presentations but may progress in worst cases to severe pneumonia leading to intensive care unit (ICU) admission and acute respiratory distress syndrome (ARDS) requiring respiratory support [1]. Since the onset original author(s) and the source, provide a link to the Creative Commons licence, and indicate if changes were made. The images or other third party material in this article are included in the article's Creative Commons licence, unless indicated otherwise in a credit line to the material. If material is not included in the article's Creative Commons licence and your intended use is not permitted by statutory regulation or exceeds the permitted use, you will need to obtain permission directly from the copyright holder. To view a copy of this licence, visit http://creativecommons.org/licenses/by/4.0/. The Creative Commons Public Domain Dedication waiver (http://creativeco mmons.org/publicdomain/zero/1.0/) applies to the data made available in this article, unless otherwise stated in a credit line to the data. 
of the pandemic, the number of COVID-19 patients has never ceased to rise and has ultimately outstripped hospital/ICU capacity in some areas particularly affected by virus spread. COVID-19 mortality, although currently decreasing, has remained dramatically high especially in patients requiring invasive mechanical ventilation [1].

An enormous research effort has been consented to describe and understand the mechanisms sustaining altered host immune response to a virus totally unknown of human immune surveillance. Many exploratory nonhypothesis-driven programs have been deployed in order to decipher immune processes at play in COVID-19. Through various flow cytometry approaches, transcriptomic strategies, functional testing and multiplex measurement of soluble mediators, most studies compared immune response between groups of COVID-19 patients with increasing severity, i.e., mild/severe/critical [2-5]. These works provided homogenous results describing that, upon hospital arrival, the most severe phenotype associated with inflammatory response (e.g., moderate plasma IL-6 elevation) and altered cellular immunity, i.e., decreased monocyte HLA-DR expression (mHLA-DR) and marked lymphopenia [6-11]. In addition, impaired type I interferons (IFN-I) activity has emerged as a contributor to the disease severity [12-15]. These latter cytokines are crucial components of innate immune response against viruses by ringing a "first alarm" bell. IFN-I have, by themselves, antiviral properties but they also induce the expression of hundreds of IFN-stimulated genes (ISG) inducing cellular antiviral activity, therefore, limiting virus spread [5].

In contrast, fewer studies focused on longitudinal immune monitoring in hospitalized COVID-19 patients, whereas their hospital stay may last for several weeks [1]. This is especially true in critically ill COVID-19 patients with ARDS who also present with the highest mortality [1]. To investigate this particular aspect of COVID-19 immune response, we monitored selected immunological parameters, including IFN $\alpha 2$ measurement and IFNstimulated genes (ISG) transcriptomic signature, in a group of 64 COVID-19 patients requiring ICU care over a 3-week period after ICU admission.

\section{Methods}

\section{Clinical study design, patient population and approval}

Between March and May 2020, critically ill patients admitted to three ICUs from academic hospital (Hospices Civils de Lyon, Lyon, France) who presented with pulmonary infection with SARS-CoV-2 confirmed by RT-PCR testing were prospectively included in the study. A flowchart describing the patient datasets used for the different analyses is provided in Additional file 1: Figure S1. Preliminary results from a subgroup of the cohort were published previously [9]. This project was part of an ongoing prospective observational clinical study (RICO, REA-IMMUNO-COVID). It was approved by ethics committee (Comité de Protection des Personnes Ile de France 1 - $\mathrm{N}^{\circ}$ IRB/IORG \#: IORG0009918) under agreement number 2020-A01079-30. This clinical study was registered at ClinicalTrials.gov (NCT04392401). The committee waived the need for written informed consent because the study was observational, with a low risk to patients, and no specific procedure, other than routine blood sampling, was required. Oral information and nonopposition to inclusion in the study were mandatory and were systematically obtained before any blood sample was drawn. This was recorded in patients' clinical files. If a patient was unable to consent directly, non-opposition was obtained from the patient's legally authorized representative and reconfirmed from the patient at the earliest opportunity. Inclusion criteria were: patients aged $>18$ years, diagnosis of COVID-19 confirmed by RTPCR testing in one respiratory sample. Inclusion criteria were (1) man or woman aged 18 or over, (2) hospitalization in ICU for SARS-CoV-2 pneumopathy, (3) first hospitalization in ICU, (4) positive diagnosis of SARS-CoV2 infection carried out by PCR or by another approved method in at least one respiratory sample, (5) sampling in the first $24 \mathrm{~h}$ after admission to ICU (D0) feasible and (6) patient or next of kin who has been informed of the terms of the study and has not objected to participating. Exclusion criteria were pregnancy, institutionalized patients, inability to obtain informed consent.

\section{Patient characteristics}

For each patient, demographics, comorbidities, time from onset of COVID-19 symptoms to ICU admission, initial presentation of the disease in ICU including the ratio of the arterial partial pressure of oxygen to the fractional inspired oxygen $\left(\mathrm{PaO}_{2} / \mathrm{FiO}_{2}\right.$ ratio $)$ at admission, antiviral therapy targeting SARS-CoV-2 and organ support were documented. Organ dysfunctions according to sequential organ failure assessment (SOFA) score (range 0-24, with higher scores indicating more severe organ failures) and simplified acute physiology score II (SAPS II; range 0-164, with higher scores indicating greater severity of illness) were documented. Patients were classified in the acute respiratory distress syndrome (ARDS) group if they were invasively ventilated and met the Berlin criteria for ARDS within the first 3 days after ICU admission [16]. Follow-up included ICU length of stay, in-hospital mortality, day-28 (D28) mortality, day-90 (D90) mortality, as well as occurrence secondary infection based on recommendation from Comité technique des infections nosocomiales et des infections liées aux soins [17]. 


\section{Blood samples}

Ethylene diamine tetraacetic acid (EDTA-)anticoagulated blood was drawn five times during the first month after ICU admission: within the first $48 \mathrm{~h}$ after admission (Day 0: D0), between 72 and $96 \mathrm{~h}$ after admission (D3), between D7 and D9 (D7), between D12 and D15 (D12) and between D20 and D25 (D20). Blood was stored at $4-8{ }^{\circ} \mathrm{C}$ and processed within $4 \mathrm{~h}$ after withdrawal. The numbers of available values for each immune parameter at each time point are presented in Additional file 1: Table S4.

\section{Cytokine measurement}

Whole blood was sampled on EDTA tubes and plasma was frozen at $-20{ }^{\circ} \mathrm{C}$ within $4 \mathrm{~h}$ following blood collection. Cytokine measurement was taken by batches after 1 freeze/thaw cycle using standardized protocols fulfilling clinical and diagnostic laboratories accreditation requirements from the International Organization for Standardization. Plasma concentrations of IL-6, TNF- $\alpha$, IFN- $\gamma$ and IL-10 were measured by Simpleplex ${ }^{\circledR}$ technology using ELLA instrument (ProteinSimple ${ }^{\circledR}$, San Jose, CA), following manufacturer's instructions. Plasma IFN 2 concentrations were determined by single-molecule Array $\left(\mathrm{SIMOA}^{\circledR}\right)$ on a HD-1 Analyzer (Quanterix) using a commercial kit for IFN- $\alpha 2$ quantification (Quanterix, Lexington, Mass).

\section{IFN-stimulated genes (ISG) score calculation}

Whole blood was collected on PAXgene blood RNA tubes (BD, Grenoble, France) for IFN signature and frozen at $-80{ }^{\circ} \mathrm{C}$ until RNA extraction. IFN score was obtained using nCounter ${ }^{\circledR}$ analysis technology (NanoString Technologies, Seattle, WA) by calculating the median of the normalized count of 6 ISGs using standardized protocols fulfilling clinical and diagnostic laboratories accreditation requirements from the International Organization for Standardization. As previously described, six interferon responsive genes were monitored: SIGLEC1 (sialic acid binding Ig like lectin 1), IFI27 (interferon alpha inducible protein27), IFI44L (interferon induced protein 44 like), IFIT1 (interferon induced protein with tetratricopeptide repeats 1), ISG15 (interferon-stimulated gene 15) and RSAD2 (radical S-adenosyl methionine domain-containing 2). Three references genes were also measured: $A C T B$ (Actin beta), HPRT1 (hypoxanthine phosphoribosyltransferase 1) and POLR2A (RNA Polymerase II Subunit A) [18].

\section{Flow cytometry}

$\mathrm{T}$ lymphocyte subpopulation immunophenotyping was performed on an automated volumetric flow cytometer from Beckman Coulter (Aquios CL) as previously described [19]. Monocyte HLA-DR expression and $B$ and NK immunophenotyping were performed using antibodies from Beckman-Coulter and BD Biosciences. The expression of monocyte HLA-DR was determined using the Anti-HLA-DR/Anti-Monocyte Quantibrite assay (BD Biosciences, San Jose, USA). A total number of antibodies bound per cell $(\mathrm{AB} / \mathrm{C})$ were quantified using calibration with a standard curve determined with $\mathrm{BD}$ Quantibrite phycoerythrin (PE) beads (BD Biosciences) as described elsewhere [20]. B and NK lymphocyte immunophenotyping was performed using lyophilized antibody panel from Beckman Coulter (Duraclone kit). Data were acquired on a Navios flow cytometer (Beckman Coulter, Hialeah, FL), and flow data were analyzed using Navios software (Beckman Coulter). Enumeration of lymphocyte subpopulations as well as mHLA-DR measurement were performed using standardized protocols fulfilling clinical and diagnostic laboratories accreditation requirements from the International Organization for Standardization.

\section{SARS-CoV-2 detection by semiquantitative PCR}

Semiquantitative values of SARS-CoV-2 viral load in upper respiratory samples at ICU admission were retrieved from clinical files for 40 patients. These results were obtained from accredited reference laboratory using RT-PCR technique with validated commercial kits $\left(\mathrm{COBAS}^{\circledR}\right.$ SARS-CoV-2, Roche Diagnostics or reference technique from Pasteur Institute) based on recommendations from the Societé Française de Microbiology after evaluation of analytical performances of the techniques [21]. Patients were classified into three groups according to SARS-CoV-2 PCR Ct values reflecting respiratory viral load at admission (1) A significant viral excretion (ct value $\leq 33$ ) subsequently divided into two groups: high viral load (Reference Ct value $<27, n=12$ ) and medium viral load (Reference Ct value $=[27-33], n=21$ ); (2) a nonsignificant viral excretion corresponding to a low viral load (Reference Ct value $=[33-37], n=7)$.

\section{Statistical analysis}

Data are presented as numbers and percentage (qualitative variables) and medians and 25th/75th percentiles (quantitative variables). Chi square or Fisher's exact test were used for qualitative variables assessment. Quantitative variables were compared with Mann-Whitney U test. For all pairs of immune parameters, Spearman's rho correlation coefficients were estimated and summarized in a correlation matrix. Kaplan-Meier survival curves were calculated in groups of patients with or without ARDS. The p-value of the log-rank test is given. The level of significance was set at $5 \%$. Data were analyzed using 
Graphpad Prism version 5.03 (Graphpad Software, La Jolla, USA).

\section{Results}

\section{Results on admission}

Sixty-four patients with confirmed pulmonary SARSCoV-2 infection admitted to three ICUs of Lyon University Hospitals (Hospices Civils de Lyon, Lyon, France) were included between March 16 and May 15, 2020. This period corresponded to the first surge in COVID19 cases in France. Clinical characteristics are presented in Table 1. Median duration of symptoms before ICU admission was 7 [4-11] days. Forty patients (63\%) presented with ARDS requiring mechanical ventilation within the first 3 days after ICU admission; 23/24 (96\%) patients without ARDS needed noninvasive ventilation (including high flow nasal oxygenotherapy) for respiratory dysfunction/failure. Flowchart and the number of samples by time point are provided in Additional file 1: Figure S1A. As assessed after 28 days, mortality in this cohort was $22 \%$ with contrasting results between patients without ARDS (no mortality) and with ARDS (35\%) (Additional file 1: Figure S1B).

On admission, critically ill COVID-19 patients presented with elevated plasma levels of both pro- and antiinflammatory cytokines (Additional file 1: Table S1). Marked lymphopenia (median $=653$ cells $/ \mu \mathrm{L}$ ) affecting all lymphocyte subsets (e.g., median CD4+T lymphocytes $=298$ cells $/ \mu \mathrm{L}$, with $\mathrm{CD} 4 / \mathrm{CD} 8$ ratio in normal range) $[10,11]$ and moderately decreased mHLA-DR (median $=11,125 \mathrm{AB} / \mathrm{C}$, control values $>13,500 \mathrm{AB} / \mathrm{C}$ ) [22] were also hallmarks of initial critically ill COVID19 patients' immune profile. Collectively, these results are in agreement with previously published values in ICU cohorts [9, 23, 24]. Interestingly, we observed that IFN-I response was induced in the majority of these patients as we measured elevated levels of plasma IFN 22 (median $=385 \mathrm{fg} / \mathrm{mL}$, control values $<20 \mathrm{fg} / \mathrm{mL}$ ) and ISG score (median $=40$, control values $<2.3$ ). These concentrations were consistent with the previously published results in cohorts of critically ill patients [12]. To note, for these inaugural immune parameters, no differences in their concentrations were observed based on delay between first symptoms and sampling time (data not shown).

When correlating immune parameters at ICU admission (Fig. 1a), significant correlations were noted between increased pro-inflammatory IL- 6 concentration and increased TNF- $\alpha$ and IL-10 levels (positive correlations), and decreased $\mathrm{T}$ lymphocyte number (negative correlation). No correlation was observed between IL-6 and IFN $\alpha 2$ levels or ISG score. In addition, initial increased IL-6 concentration was correlated with initial severity as measured by sepsis-related organ failure assessment (SOFA) score and with intensity of pulmonary dysfunction as measured by the ratio between partial pressure of oxygen in arterial blood $\left(\mathrm{PaO}_{2}\right)$ and fraction of inspired oxygen $\left(\mathrm{FiO}_{2}\right)\left(\mathrm{PaO}_{2} / \mathrm{FiO}_{2}\right.$ ratio, Fig. 1b). Finally, when classifying patients into three groups according to semiquantitative levels of viremia measured in upper respiratory samples, the group of patients with the highest viral load presented not only with higher plasma IL-6 concentrations at admission but also with a higher SOFA score and increased mortality (33\%, Additional file 1: Figure S2). This suggests that the immune response is positively associated with viral burden and that failure to resolve both aspects may underlie severity [8].

\section{Comparison between ARDS and non-ARDS patients}

Considering the significant difference between ARDS and non-ARDS groups regarding D28 mortality (Additional file 1: Figure $\mathrm{S} 1 \mathrm{~B}$ ), we then compared immune profiles between these 2 groups of patients (Fig. 2, Additional file 1: Table S2). As most non-ARDS patients presented with a short ICU stay of less than 1 week, we focused on the first three time points after ICU admission. NonARDS patients presented with a different immune profile than patients with ARDS, including higher lymphocytes count and mHLA-DR expression and lower cytokine levels and severity scores. Except for IL- 6 and TNF- $\alpha$, these differences persisted over the first week of followup (Fig. 2). We observed no difference between ARDS and non-ARDS patients regarding plasma IFN $\alpha 2$ measurements or ISG score (Fig. 2). The correlation between these two parameters was statistically significant $(r=0.7$, $p<0.0001)$. In addition, we observed a rapid decrease of IFN-I markers after ICU admission in both groups. This shows that IFN-I response was induced in critically ill COVID-19 patients and efficient in activating downstream genes. However, the intensity of this response was not impacted by the development of pulmonary dysfunction or disease severity upon ICU admission.

\section{Comparison between survivors and non-survivors among ARDS patients}

We next focused on ARDS patients who presented with a prolonged ICU length of stay to evaluate immune profiles in regard with D28 mortality during a 3-week follow-up (Fig. 3, Additional file 1: Table S3). Clinical characteristics of survivors and non-survivors are presented in Table 2. Surprisingly, we did not observe any difference between survivors and non-survivors regarding the majority of immune parameters. In this cohort, survivors and non-survivors presented with apparent common immune responses mixing gradual return to normal ranges of cellular markers (mHLA-DR, 
Table 1 Clinical characteristics of critically ill patients with COVID-19 at ICU admission

\begin{tabular}{|c|c|c|c|c|}
\hline & All patients $(n=64)$ & $\operatorname{ARDS}(n=40)$ & No ARDS $(n=24)$ & $p$ value \\
\hline \multicolumn{5}{|l|}{ Demographics } \\
\hline Age & $65[52-72]$ & $66[57-72]$ & $55[43-72]$ & 0.0738 \\
\hline Gender & $51(80 \%)$ & $36(90 \%)$ & $15(63 \%)$ & 0.0116 \\
\hline Body mass index $\left(\mathrm{kg} / \mathrm{m}^{2}\right)$ & $28[26-32]$ & $29[26-34]$ & $28[25-30]$ & 0.2124 \\
\hline Body mass index $>30$ kg/m² & $23(36 \%)$ & $17(43 \%)$ & $6(25 \%)$ & 0.1874 \\
\hline \multicolumn{5}{|l|}{ Comorbidities } \\
\hline Diabetes & $16(25 \%)$ & $11(25 \%)$ & $5(21 \%)$ & 0.7693 \\
\hline Comorbidities & & & & 0.2979 \\
\hline 0 & $36(56 \%)$ & $20(50 \%)$ & $16(67 \%)$ & \\
\hline$\geq 1$ & $28(44 \%)$ & $20(50 \%)$ & $8(33 \%)$ & \\
\hline Charlson score & $0[0-2]$ & $1[0-2]$ & $0[0-1]$ & 0.0002 \\
\hline \multicolumn{5}{|l|}{ Admission symptoms } \\
\hline Delay between first symptoms (Days) & $7[4-11]$ & $7[4-10]$ & $8.5[7-11]$ & 0.2055 \\
\hline Fever & $52(81 \%)$ & $34(85 \%)$ & $18(75 \%)$ & 0.3414 \\
\hline Cough & $42(66 \%)$ & $24(60 \%)$ & $18(75 \%)$ & 0.2814 \\
\hline Dyspnea & $40(63 \%)$ & $28(70 \%)$ & $12(50 \%)$ & 0.1208 \\
\hline Diarrhea & $18(28 \%)$ & $10(25 \%)$ & $8(33 \%)$ & 0.5691 \\
\hline Diffuse pain & $13(20 \%)$ & $7(18 \%)$ & $6(25 \%)$ & 0.5300 \\
\hline Altered general status & $44(69 \%)$ & $24(60 \%)$ & $20(83 \%)$ & 0.0584 \\
\hline Other & $28(44 \%)$ & $16(40 \%)$ & $12(50 \%)$ & \\
\hline \multicolumn{5}{|l|}{ Severity scores } \\
\hline SOFA score & $4[2-8]$ & $8[4-9]$ & $2[2,3]$ & $<0.0001$ \\
\hline SAPS II score & $34[26-45]$ & $40[32-54]$ & $27[21-33]$ & $<0.0001$ \\
\hline $\mathrm{PaO}_{2} / \mathrm{FiO}_{2}$ at admission & 145 [92-191] & $132[95-166]$ & 230 [83-298] & 0.0152 \\
\hline \multicolumn{5}{|l|}{ Antiviral therapy } \\
\hline Hydroxychloroquine & $35(55 \%)$ & $20(50 \%)$ & $15(63 \%)$ & 0.4381 \\
\hline Lopinavir/ritonavir & $5(8 \%)$ & $3(8 \%)$ & $2(8 \%)$ & $>0.9999$ \\
\hline Lopinavir/ritonavir + interferon $\beta$ & $5(8 \%)$ & $4(10 \%)$ & $1(4 \%)$ & $>0.9999$ \\
\hline Remdesivir & $1(2 \%)$ & $1(3 \%)$ & $0(0 \%)$ & 0.5238 \\
\hline \multicolumn{5}{|l|}{ Organ support } \\
\hline Mechanical ventilation & $63(98 \%)$ & $40(100 \%)$ & $23(96 \%)$ & 0.3750 \\
\hline Noninvasive ventilation & $23(36 \%)$ & $0(0 \%)$ & $23(100 \%)$ & $<0.0001$ \\
\hline Invasive ventilation & $40(63 \%)$ & $40(100 \%)$ & $0(0 \%)$ & $<0.0001$ \\
\hline Vasoactive drugs & $24(38 \%)$ & $24(60 \%)$ & $0(0 \%)$ & $<0.0001$ \\
\hline Renal replacement therapy & 15 (23\%) & $15(38 \%)$ & $0(0 \%)$ & 0.0004 \\
\hline \multicolumn{5}{|l|}{ Follow-up } \\
\hline Days in ICU & $10[4-30]$ & $21[11-58]$ & $4[2-7]$ & 0.003 \\
\hline Days in hospital & $21[11-56]$ & 33 [19-71] & 13 [8-21] & $<0.0001$ \\
\hline Hospital mortality & $19(30 \%)$ & $19(48 \%)$ & $0(0 \%)$ & $<0.0001$ \\
\hline Day-28 mortality & $14(22 \%)$ & $14(35 \%)$ & $0(0 \%)$ & $<0.0001$ \\
\hline Day-90 mortality & $19(30 \%)$ & $19(48 \%)$ & $0(0 \%)$ & $<0.0001$ \\
\hline Secondary infections & $32(50 \%)$ & $30(75 \%)$ & $2(8 \%)$ & $<0.0001$ \\
\hline
\end{tabular}

The results are shown as medians and interquartile ranges [Q1-Q3] for continuous variables or numbers and percentage for categorical variables. Patients were separated into two groups based on presence of acute respiratory distress syndrome (ARDS) according to Berlin definition during the first $72 \mathrm{~h}$ after admission. Sepsisrelated organ failure assessment (SOFA) and simplified acute physiology score II (SAPS II) scores were calculated during the first $24 \mathrm{~h}$ after intensive care unit (ICU) admission. Data were compared using nonparametric Mann-Whitney test for continuous variables or Fisher's exact test for categorical variables

$p$ values inferior to 0.05 are highlighted in bold

lymphocyte count) and progressive decrease of cytokines levels including IFN 2 (and related ISG score). Only plasma TNF- $\alpha$ presented with a specific kinetic as it was the only cytokine to slowly rise during 


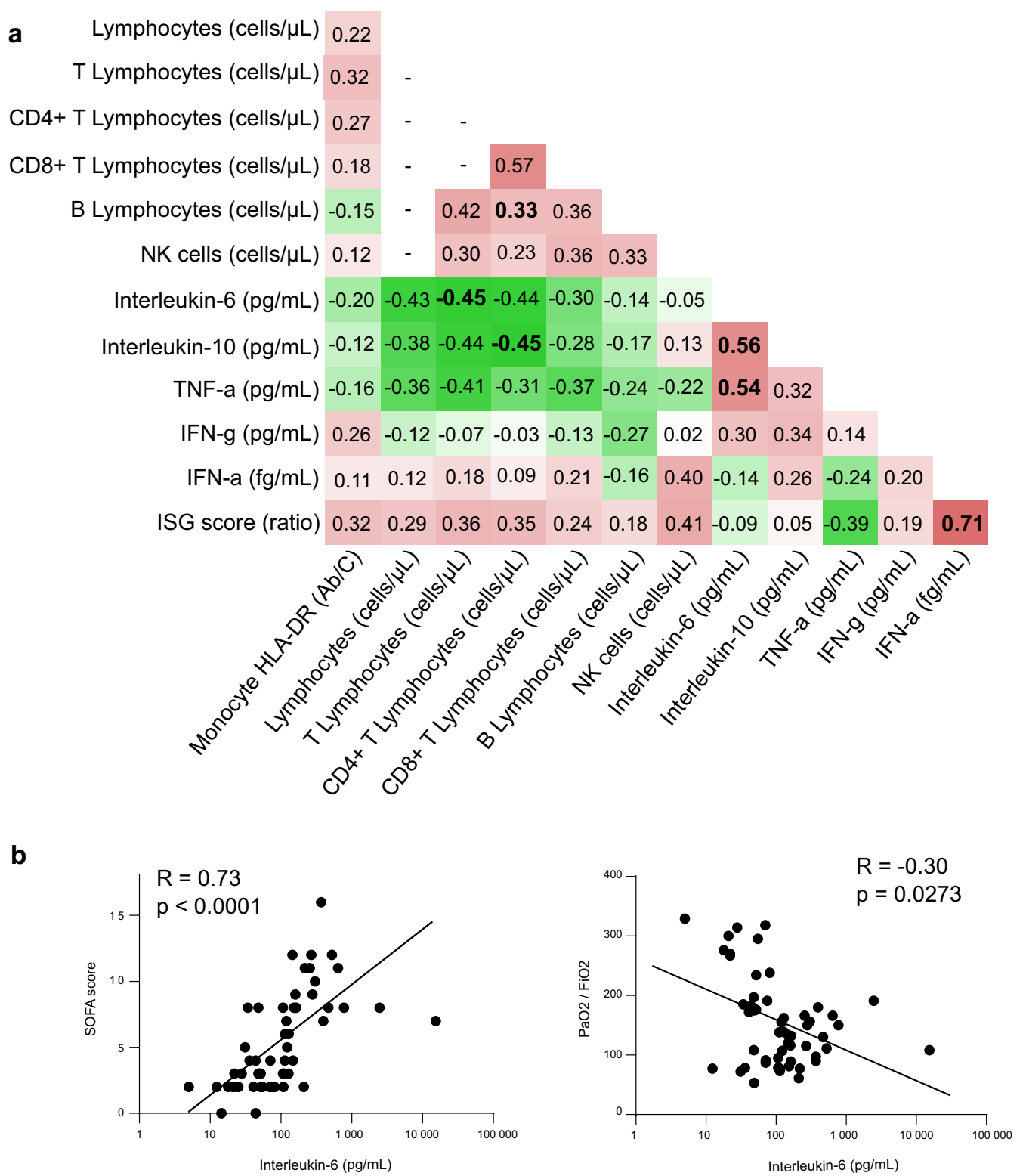

Fig. 1 Correlation matrix of immune parameters at ICU admission. Immune parameters were measured at inclusion in 64 critically ill patients with COVID-19, and correlations were calculated using Spearman correlation tests. a Results are presented as a correlation matrix. Spearman correlation coefficients are plotted. Cells were colored according to the strength and trend of correlations (shades of red = positive, shades of green $=$ negative correlations). Coefficients with a $p$ value below 0.005 were highlighted in bold and considered significant after correction for multiple testing. Correlation results for non-independent parameters (i.e., lymphocyte subpopulations) are not presented. b Correlations between plasma interleukin-6 concentration at inclusion and sepsis-related organ failure (SOFA) score or $\mathrm{PaO}_{2} / \mathrm{FiO}_{2}$ ratio measured during first $24 \mathrm{~h}$ after admission are shown $(n=58)$. Corresponding logarithmic trendlines are shown

the follow-up. Moreover, its levels were significantly higher in non-surviving patients compared with survivors at all time points. This may reflect the high rates of secondary infections occurring in ARDS COVID19 patients [25-27]. For example, in this cohort, 81\% of survivors and $64 \%$ of non-survivors ARDS patients developed a secondary infection during their ICU stay. The constant elevation of neutrophil count during the whole monitoring in ARDS patients probably also reflect such re-stimulation of the immune system in response to secondary infectious challenges (Additional file 1: Table S3). Last, we observed that nonsurvivors exhibited a significant rise in CD4/CD8 ratio compared with survivors (Fig. 3). This was mainly due 


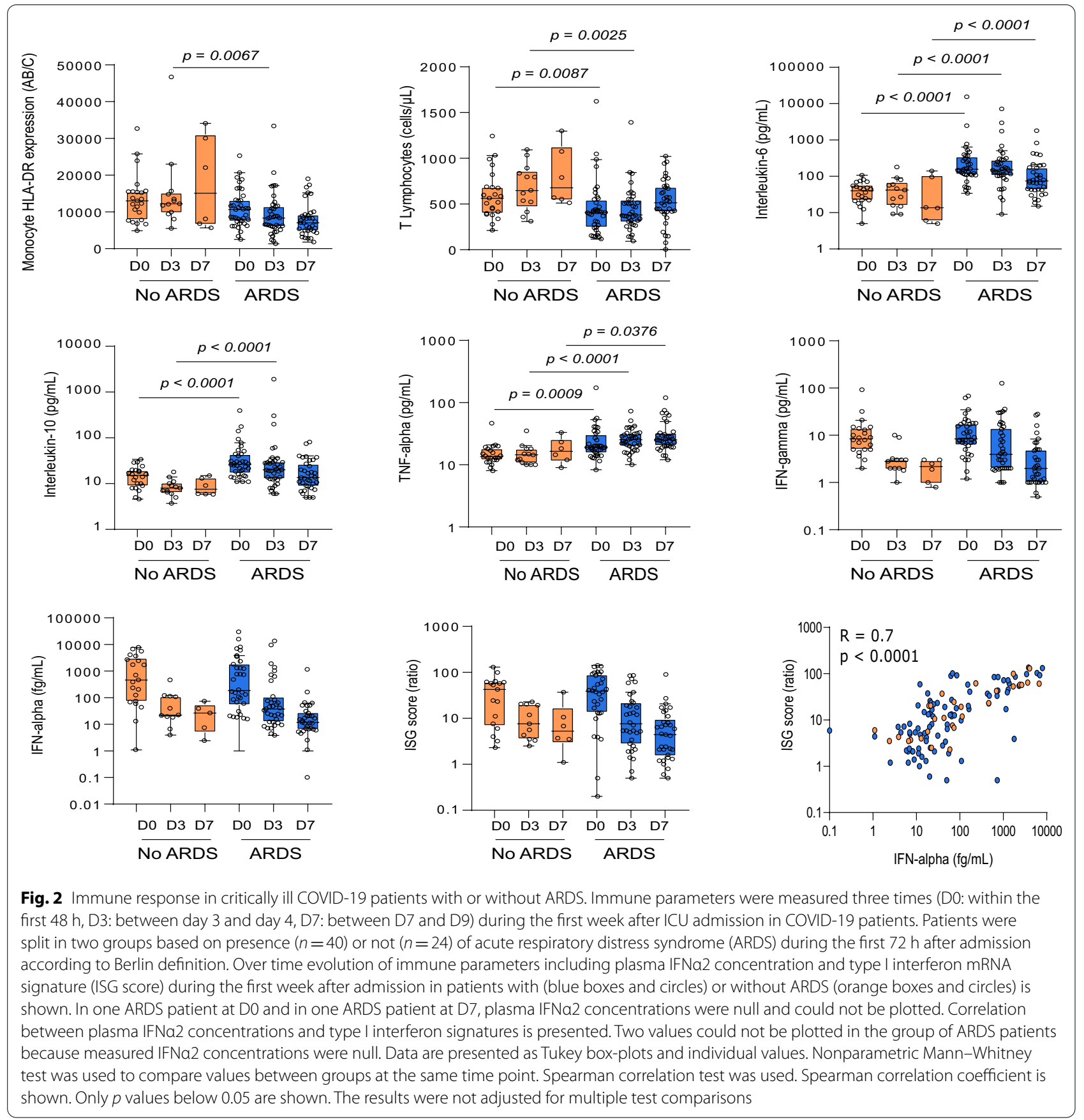

to the slower recovery of $\mathrm{CD} 8+\mathrm{T}$ cell numbers in this group (Additional file 1: Table S3).

\section{Discussion}

Collectively, the present results provide an unbiased description of COVID-19 immuno-inflammatory derangements in critically ill COVID-19 patients focusing on ARDS patients who exhibit the highest mortality. We noted that, upon ICU admission, immune response to SARS-CoV-2 infection presents with similarities with bacterial sepsis $[28,29]$. These include (1) severe lymphopenia affecting all lymphocyte subsets, (2) moderately decreased mHLA-DR and (3) moderately increased plasma cytokine levels showing at the same time both inflammatory (IL-6) and immunosuppressive (IL-10) responses. In addition, we noticed increased plasma IFN 22 levels and ISG score indicating the occurrence of an IFN-I response. This agrees with increased CD169 


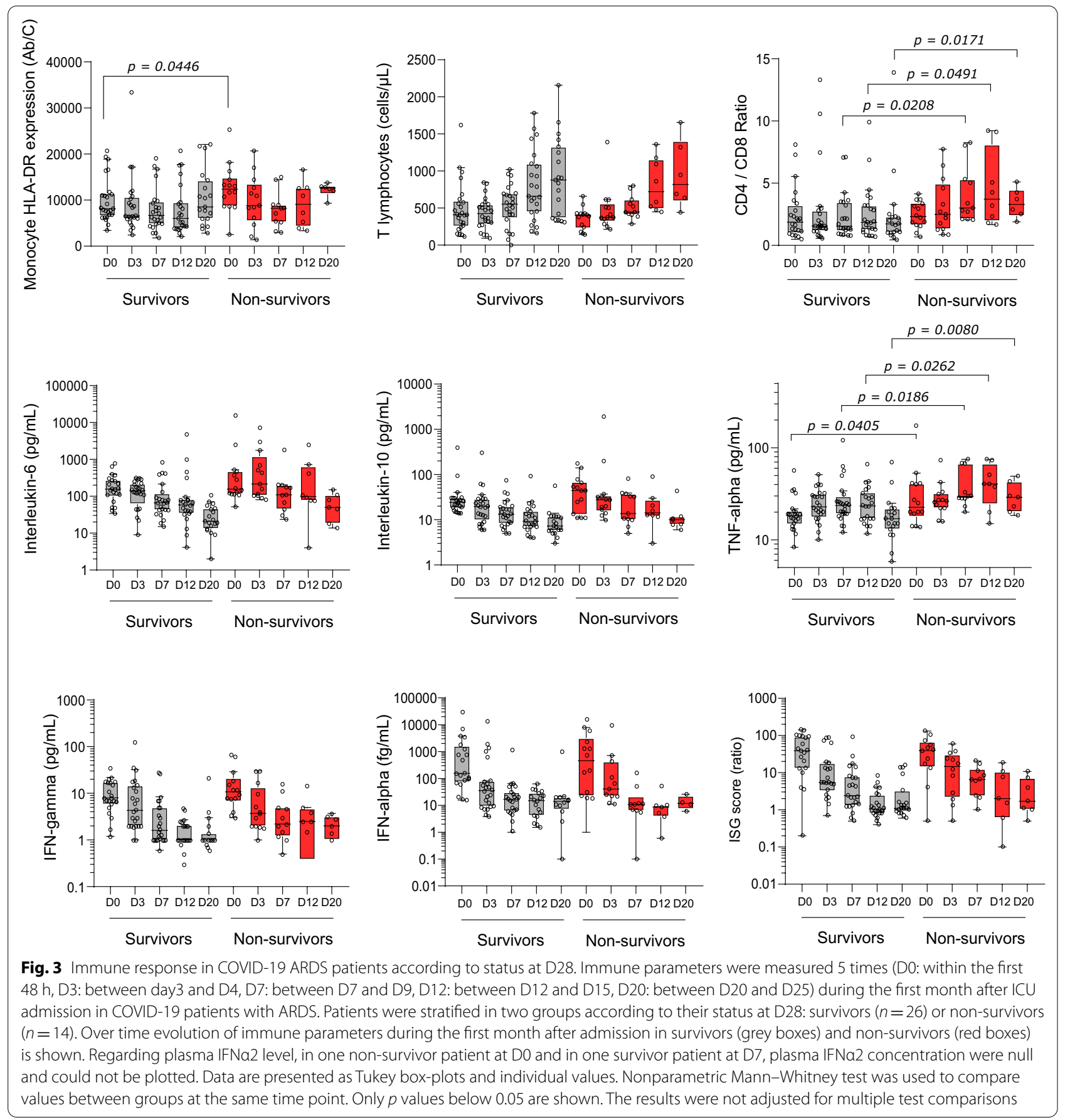

expression on monocytes (aka siglec-1, one of the six genes of ISG) in COVID-19 patients upon ICU admission [30]. Thus, the present results do not support a potentially altered IFN-I response in the majority of COVID19 patients upon ICU admission. However, as IFN 22 concentrations reported elsewhere were higher in less severe/moderate patients (between 1000 and $5000 \mathrm{fg} /$ $\mathrm{mL}$ ) [12]; we cannot exclude that the incapacity to mount a full type-I IFN response immediately following SARSCoV2 infection in some patients may have led to their worsening and ultimately to ICU admission.

These abnormalities (along with decreased plasmacytoid cells $[12,14,31])$ are reminiscent of the process of age-acquired immunosuppression (also called immunosenescence) observed in elderly people who are, by far, the primary victims of COVID-19. We may hypothesize 
Table 2 Clinical characteristics of patients with COVID-19 with ARDS according to status at D28

\begin{tabular}{|c|c|c|c|}
\hline & Survivors $(n=26)$ & Non-survivors $(n=14)$ & $p$ value \\
\hline \multicolumn{4}{|l|}{ Demographics } \\
\hline Age & $65[55-70]$ & $67[58-78]$ & 0.3076 \\
\hline Gender & $23(88 \%)$ & $13(93 \%)$ & $>0.9999$ \\
\hline Body mass index $\left(\mathrm{kg} / \mathrm{m}^{2}\right)$ & 30 [27-35] & $28[24-30]$ & 0.0960 \\
\hline Body mass index $>30 \mathrm{~kg} / \mathrm{m}^{2}$ & $14(54 \%)$ & $3(21 \%)$ & 0.0921 \\
\hline Delay between first symptoms (Days) & $7[4-12]$ & $5[4-9]$ & 0.1909 \\
\hline \multicolumn{4}{|l|}{ Comorbidities } \\
\hline Comorbidities & & & 0.3202 \\
\hline 0 & $15(58 \%)$ & $5(36 \%)$ & \\
\hline$\geq 1$ & $11(42 \%)$ & $9(64 \%)$ & \\
\hline Charlson score & $0[0-2]$ & $2[0-2]$ & 0.1281 \\
\hline \multicolumn{4}{|l|}{ Severity scores } \\
\hline SOFA score & $8[3-10]$ & $8[4-8]$ & 0.7937 \\
\hline SAPS II score & $41[31-52]$ & $40[33-59]$ & 0.5893 \\
\hline $\mathrm{PaO}_{2} / \mathrm{FiO}_{2}$ at admission & $150[94-169]$ & $116[94-162]$ & 0.2993 \\
\hline ARDS severity mild & $1(4 \%)$ & $1(7 \%)$ & $>0.9999$ \\
\hline Moderate & $18(69 \%)$ & $9(64 \%)$ & $>0.9999$ \\
\hline Severe & $7(27 \%)$ & $4(29 \%)$ & $>0.9999$ \\
\hline \multicolumn{4}{|l|}{ Follow-up } \\
\hline Days in ICU & $40[16-76]$ & $11[6-20]$ & 0.0008 \\
\hline Days in hospital & 64 [38-77] & $15[7-20]$ & $<0.0001$ \\
\hline Secondary infections & $21(81 \%)$ & $9(64 \%)$ & 0.2777 \\
\hline
\end{tabular}

The results are shown as medians and interquartile ranges [Q1-Q3] for continuous variables or numbers and percentage for categorical variables. COVID-19 patients with ARDS were separated in two groups based on status at D28 after admission. Sepsis-related organ failure assessment (SOFA) and simplified acute physiology score II (SAPS II) scores were calculated during the first $24 \mathrm{~h}$ after admission. Patients were classified in ARDS severity groups according to Berlin criteria. ICU: intensive care unit. Data were compared using nonparametric Mann-Whitney test for continuous variables or Fisher's exact test for categorical variables

$p$ values inferior to 0.05 are highlighted in bold

that evolution of COVID-19 toward increasing severity in this population of old patients is mainly a consequence of this altered immune status [32-35]. For example, previous studies showed the negative correlation between lymphocytes count and pulmonary viral load [36, 37] while, elsewhere, the magnitude of pulmonary viral load was repeatedly associated with increased mortality $[8,38-43]$. In the present work, this is also illustrated by the association between nasopharyngeal viral load and increased mortality, SOFA and IL-6 levels upon admission.

Our results emphasize that ARDS occurrence appears to be an important driver of mortality during COVID-19 progression in ICU patients [1]. In the present cohort, mortality occurred only in the group of patients with ARDS while all ICU patients without ARDS were released alive from the ICU. This observation makes a strong priority for avoiding progression toward ARDS in COVID-19 patients. After ICU admission, inflammatory response as well as IFN-I activity progressively normalized while immunological cellular parameters remained below references ranges. Thus, as seen in bacterial sepsis, ARDS occurrence in COVID19 patients may secondarily amplify COVID-19-induced immune alterations either by direct cytotoxic effect and/or by negative anti-inflammatory feedbacks $[28,29,32]$. This leads to the development of a torpid immunosuppressed status in ARDS patients which may last several weeks [44].

In such critically ill patients with prolonged ICU stays, this immunosuppressed status presents with deleterious consequences. First, it probably participates in the long duration necessary to eradicate SARS-CoV-2 from the lung in invasively ventilated patients as described elsewhere [43]. For example, it was shown that the viral shedding in lower respiratory tract lasted almost 30 days in median in critically ill COVID-19 patients [38, 42]. Second, it most likely contributes to the very high rates of nosocomial infections reported not only in the present study (50\%) but also in many others $[26,27,45]$. In particular, COVID-19 is characterized by astonishing elevated rates of secondary aspergillosis [46-48], a fungal disease usually seen in the most immunosuppressed patients. Such secondary infectious events may explain 
the persistently elevated TNF- $\alpha$ levels accompanied by increased neutrophil count in COVID-19 patients with ARDS and in non-survivors [25]. This suggests that in this second step of the disease, i.e., once ARDS occurred, immunostimulation could represent a sound approach to try to promote immune recovery and prevent secondary bacterial and fungal infections.

The most striking result of the present study was to observe similar altered immune response in both surviving and non-surviving ARDS patients over a 3-week follow-up. Thus, the duration of this COVID-19 induced immunosuppressed status remains to be defined. While a recent study observed that the onset of $\mathrm{T}$ cell recovery in COVID-19 ICU patients with ARDS started on day 35 [49], the 3-week ICU follow-up performed in this study may have been too short to distinguish immune trajectories according to outcomes in COVID-19 ARDS patients. Of note, in this latter work [49], patients with unfavorable outcome presented with increased CD4/CD8 ratio as observed in the present study. We may thus hypothesize that, in ARDS patients, the lack of CD8+T lymphocyte recovery could be a poor prognosis factor $[35,50]$.

Being exploratory, our work presents with limitations. First, SARS-CoV2 viral load could not be regularly monitored. Further studies should include strict quantitative evaluation of viral load with standardized tools throughout the monitoring to decipher the duration of viral persistence in the lung and its correlation with immune response and outcomes. Second, functional testing of immune cells was not performed. If available, this should be incorporated in addition with phenotypic markers of immune response [51-53]. This is especially true regarding $\mathrm{CD} 8+\mathrm{T}$ cell functionality in response to SARS-CoV-2 peptides that may help to understand which comes first: CD8 efficiency (and recovery) or virus disappearance. Three, we did not include less severe COVID-19 cohorts in order to explore (with single-molecule array-SIMOAtechnology) the magnitude of IFN-I response in patients who correctly eradicated the virus. Last, at the time of first COVID-19 surge in France (corresponding to the patients reported here), optimized care protocols (oxygen, heparin, dexamethasone) were not applied. Therefore, some observations need to be confirmed under the angle of current clinical practice.

\section{Conclusions}

In sum, upon patients' admission to the ICU, pulmonary virus spread is accompanied by an inflammatory response characterized by moderately increased circulating levels of typical inflammatory cytokines (e.g., IL-6 levels usually $<100 \mathrm{pg} / \mathrm{mL}$ ) [54-57]. At this stage, no obvious observation of altered IFN-I response could be reported. If not appropriately controlled by the immune system [33], virus replication in lungs and related inflammation may progressively lead to ARDS which appears to be one driver of mortality. Following this acute response leading to pulmonary dysfunction, inflammatory response rapidly declined. As observed in bacterial sepsis $[28,29]$, patients subsequently present with a marked delayed immunosuppression. This state of immunosuppression likely prevents efficient virus eradication from the lung, facilitates virus spread outside lungs as illustrated by the deleterious association of persistent viremia and mortality [58-62]. This also probably favors the occurrence of frequent secondary infections with opportunistic pathogens [26, 27, 47]. All these elements explain the long ICU stay of invasively ventilated COVID-19 patients. In the current 3-week monitoring of ARDS patients, we did not identify any immunological parameter that significantly associated with mortality. Thus, the better understanding of the mechanisms which finally permit survival after several weeks in ICU is a crucial issue for next studies.

\section{Abbreviations}

$A B / C$ : Numbers of antibodies bound per cell; ARDS: Acute respiratory distress syndrome; HLA-DR: Human leukocyte antigen-DR; ICU: Intensive care unit; ISG: Interferon-stimulated genes; SAPS II: Simplified acute physiology score II; SOFA: Sequential organ failure assessment score; $\mathrm{PaO}_{2} / \mathrm{FiO}_{2}$ ratio: Ratio of the arterial partial pressure of oxygen to the fractional inspired oxygen.

\section{Supplementary Information}

The online version contains supplementary material available at https://doi. org/10.1186/s13054-021-03558-w.

Additional file 1. Table S1. Immune response in critically ill COVID-19 patients, Table S2. Immune response in critically ill COVID-19 patients with or without ARDS, Table S3. Immune response in critically COVID-19 patients with ARDS according to status at D28, Table S4. Numbers of available values for each immune parameter at each time point; Figure S1. Flowchart and Survival Curves and Figure S2. Impact of viral load at admission.

\section{Acknowledgements}

This work was supported by funds from the Hospices Civils de Lyon, Fondation $\mathrm{HCL}$ and Claude Bernard Lyon 1 University/Région Auvergne Rhône-Alpes. The authors would like to thank the clinical teams from all ICUs in HCL who were involved in this project while dedicated to their clinical duties during COVID19 pandemic as well as patients and their families who agreed to participate to this clinical study. The authors declare no competing financial interest. $\mathrm{K}$ Brengel-Pesce is bioMérieux employee. This private company had no role in the study design, result analysis and decision to publish this study.

The names of the individual members of the RICO study group as listed below should be searchable through their individual PubMed records:

Hospices Civils de Lyon, Lyon-Sud University Hospital, Immunology Laboratory: Remi Pescarmona, Lorna Garnier, Christine Lombard, Magali Perret, Marine Villard

Joint Research Unit HCL-bioMérieux: Valérie Cheynet, Filippo Conti

Centre d'Investigation Clinique de Lyon (CIC 1407 Inserm): Marie Groussaud, Marielle Buisson, Laetitia Itah, Inesse Boussaha

Hospices Civils de Lyon, Edouard Herriot Hospital, Immunology Laboratory: Françoise Poitevin-Later, Christophe Malcus, Morgane Gossez 
RICO clinical investigators: Florent Wallet, Marie-Charlotte Delignette, Frederic Dailler

Hospices Civils de Lyon, Edouard Herriot Hospital, Medical intensive Care Department: Marie Simon, Auguste Dargent, Pierre-Jean Bertrand, Neven Stevic, Marion Provent

Hospices Civils de Lyon, Edouard Herriot Hospital, Anesthesia and Critical Care Medicine Department: Laurie Bignet, Valérie Cerro

Hospices Civils de Lyon, Croix-Rousse University Hospital, Medical intensive Care Department: Jean-Christophe Richard, Laurent Bitker, Mehdi Mezidi, Loredana Baboi

\section{Authors' contributions}

$F V, G M, L A, T R, M C$ and $A C L$ designed the study, analyzed results, discussed findings and wrote the manuscript. RC, SV, KBP and JSC performed immune monitoring experiments and viral load monitoring. RC, SV and JSC analyzed results, discussed findings and wrote the manuscript. CM, PA and HY collected clinical samples, discussed findings and wrote the manuscript. CA analyzed results and discussed findings. All authors read and approved the final manuscript.

\section{Funding}

This work was supported by funds from the Hospices Civils de Lyon, Fondation HCL and Claude Bernard Lyon 1 University/Région Auvergne Rhône-Alpes. Reagents for type-I IFN dosage by SIMOA were provided by bioMérieux. This private company had no role in the study design, result analysis and decision to publish this study.

\section{Availability of data and materials}

The datasets analyzed during the current study are available from the corresponding author on reasonable request.

\section{Declarations}

\section{Ethics approval and consent to participate}

This project was part of an ongoing prospective observational clinical study (RICO, REA-IMMUNO-COVID). It was approved by ethics committee (Comité de Protection des Personnes lle de France 1 - NoIRB/IORG \#: IORG0009918) under agreement number 2020-A01079-30. This clinical study was registered at ClinicalTrials.gov (NCT04392401). The committee waived the need for written informed consent because the study was observational, with a low risk to patients, and no specific procedure, other than routine blood sampling, was required. Oral information and non-opposition to inclusion in the study were mandatory and were systematically obtained before any blood sample was drawn. This was recorded in patients' clinical files. If a patient was unable to consent directly, non-opposition was obtained from the patient's legally authorized representative and reconfirmed from the patient at the earliest opportunity

\section{Consent for publication}

Not applicable.

\section{Competing interests}

The authors declare no competing financial interests in relation to the work.

\section{Author details}

${ }^{1}$ Immunology Laboratory, Hôpital E. Herriot - Hospices Civils de Lyon, 5 place d'Arsonval, 69437 Lyon Cedex 03, France. ${ }^{2}$ Joint Research Unit HCL-bioMérieux, EA 7426"Pathophysiology of Injury-Induced Immunosuppression" (Université Claude Bernard Lyon 1 - Hospices Civils de Lyon - bioMérieux), 69003 Lyon, France. ${ }^{3}$ Centre International de Recherche en Infectiologie (CIRI), Inserm U1111, CNRS, UMR5308, Ecole Normale Supérieure de Lyon, Université Claude, Bernard-Lyon 1, Lyon, France. ${ }^{4}$ Medical Intensive Care Department, Edouard Herriot Hospital, Hospices Civils de Lyon, 69437 Lyon, France. ${ }^{5}$ Anesthesia and Critical Care Medicine Department, Edouard Herriot Hospital, Hospices Civils de Lyon, 69437 Lyon, France. ${ }^{6}$ Immunology Laboratory, Lyon-Sud University Hospital, Hospices Civils de Lyon, 69495 Pierre-Bénite, France. ${ }^{7}$ Medical Intensive Care Department, Croix-Rousse University Hospital, Hospices Civils de Lyon, 69004 Lyon, France. ${ }^{8}$ Centre d'Investigation Clinique de Lyon (CIC 1407 Inserm), Hospices Civils de Lyon, 69677 Lyon, France. ${ }^{9}$ Virology Laboratory, Croix-Rousse University Hospital, Hospices Civils de Lyon,
69004 Lyon, France. ${ }^{10}$ Immunology Laboratory, Lyon-Sud University Hospital, Hospices Civils de Lyon, Lyon, France. ${ }^{11}$ Joint Research Unit HCL-bioMérieux, Lyon, France. ${ }^{12}$ Centre d'Investigation Clinique de Lyon (CIC 1407 Inserm), Lyon, France. ${ }^{13}$ Immunology Laboratory, Edouard Herriot Hospital, Hospices Civils de Lyon, Lyon, France. ${ }^{14}$ RICO Clinical Investigators, Lyon, France.

${ }^{15}$ Medical Intensive Care Department, Edouard Herriot Hospital, Hospices Civils de Lyon, Lyon, France. ${ }^{16}$ Anesthesia and Critical Care Medicine Department, Edouard Herriot Hospital, Hospices Civils de Lyon, Lyon, France. ${ }^{17}$ Medical Intensive Care Department, Croix-Rousse University Hospital, Hospices Civils de Lyon, Lyon, France.

Received: 27 January 2021 Accepted: 30 March 2021

Published online: 12 April 2021

\section{References}

1. The C-ICUI Network C-IGobotR. Clinical characteristics and day-90 outcomes of 4244 critically ill adults with COVID-19: a prospective cohort study. Intensive Care Med. 2021:47(1):60-73.

2. Laing AG, Lorenc A, Del Molino Del Barrio I, Das A, Fish M, Monin L, et al. A dynamic COVID-19 immune signature includes associations with poor prognosis. Nat Med. 2020;26(10):1623-35.

3. Arunachalam PS, Wimmers F, Mok CKP, Perera R, Scott M, Hagan T, et al. Systems biological assessment of immunity to mild versus severe COVID19 infection in humans. Science. 2020;369(6508):1210-20.

4. Silvin A, Chapuis N, Dunsmore G, Goubet AG, Dubuisson A, Derosa L, et al. Elevated calprotectin and abnormal myeloid cell subsets discriminate severe from mild COVID-19. Cell. 2020;182(6):1401-18.

5. Schulte-Schrepping J, Reusch N, Paclik D, Bassler K, Schlickeiser S, Zhang $B$, et al. Severe COVID-19 is marked by a dysregulated myeloid cell compartment. Cell. 2020;182(6):1419-40.

6. Del Valle DM, Kim-Schulze S, Huang HH, Beckmann ND, Nirenberg S, Wang B, et al. An inflammatory cytokine signature predicts COVID-19 severity and survival. Nat Med. 2020;26(10):1636-43.

7. Aziz M, Fatima R, Assaly R. Elevated interleukin-6 and severe COVID-19: a meta-analysis. J Med Virol. 2020;92(11):2283-5.

8. Lucas C, Wong P, Klein J, Castro TBR, Silva J, Sundaram M, et al. Longitudinal analyses reveal immunological misfiring in severe COVID-19. Nature. 2020;584(7821):463-9.

9. Monneret G, Cour M, Viel S, Venet F, Argaud L. Coronavirus disease 2019 as a particular sepsis: a 2-week follow-up of standard immunological parameters in critically ill patients. Intensive Care Med. 2020;46(9):1764-5.

10. Huang I, Pranata R. Lymphopenia in severe coronavirus disease-2019 (COVID-19): systematic review and meta-analysis. J Intensive Care. 2020;8:36.

11. Huang W, Berube J, McNamara M, Saksena S, Hartman M, Arshad T, et al. Lymphocyte subset counts in COVID-19 patients: a meta-analysis. Cytometry A. 2020;97(8):772-6.

12. Hadjadj J, Yatim N, Barnabei L, Corneau A, Boussier J, Smith N, et al. Impaired type I interferon activity and inflammatory responses in severe COVID-19 patients. Science. 2020;369(6504):718-24.

13. Bastard $P$, Rosen LB, Zhang Q, Michailidis E, Hoffmann HH, Zhang $Y$, et al. Autoantibodies against type I IFNs in patients with life-threatening COVID-19. Science. 2020;370(6515):eabd4585.

14. Acharya D, Liu G, Gack MU. Dysregulation of type I interferon responses in COVID-19. Nat Rev Immunol. 2020:20(7):397-8.

15. Trouillet-Assant S, Viel S, Gaymard A, Pons S, Richard JC, Perret M, et al. Type I IFN immunoprofiling in COVID-19 patients. J Allergy Clin Immunol. 2020;146(1):206-8.

16. Ferguson ND, Fan E, Camporota L, Antonelli M, Anzueto A, Beale R, et al. The Berlin definition of ARDS: an expanded rationale, justification, and supplementary material. Intensive Care Med. 2012;38(10):1573-82.

17. Comité technique des infections nosocomiales et des infections liées aux soins (CTINILS) du Ministère de la santé dljeds. https://solidarites-sante. gouv.fr/IMG/pdf/rapport_vcourte.pdf. May 2007.

18. Pescarmona R, Belot A, Villard M, Besson L, Lopez J, Mosnier I, et al. Comparison of RT-gPCR and Nanostring in the measurement of blood interferon response for the diagnosis of type I interferonopathies. Cytokine. 2019;113:446-52. 
19. Gossez M, Malcus C, Demaret J, Frater J, Poitevin-Later F, Monneret $\mathrm{G}$. Evaluation of a novel automated volumetric flow cytometer for absolute CD4+ T lymphocyte quantitation. Cytom B Clin Cytom. 2017;92(6):456-64.

20. Demaret J, Walencik A, Jacob MC, Timsit JF, Venet F, Lepape A, et al. Interlaboratory assessment of flow cytometric monocyte HLA-DR expression in clinical samples. Cytom B Clin Cytom. 2013;84(1):59-62.

21. Microbiologie SFd. 2020. https://www.sfm-microbiologie.org/wp-conte nt/uploads/2021/01/Avis-SFM-valeur-Ct-excre\%CC\%81tion-virale-_-Versi on-def-14012021_V4.pdf.

22. Benlyamani I, Venet F, Coudereau R, Gossez M, Monneret G. Monocyte HLA-DR measurement by flow cytometry in COVID-19 patients: an interim review. Cytom A. 2020;97(12):1217-21.

23. Jeannet R, Daix T, Formento R, Feuillard J, Francois B. Severe COVID-19 is associated with deep and sustained multifaceted cellular immunosuppression. Intensive Care Med. 2020;46(9):1769-71.

24. Kox M, Frenzel T, Schouten J, van de Veerdonk FL, Koenen H, Pickkers P, et al. COVID-19 patients exhibit less pronounced immune suppression compared with bacterial septic shock patients. Crit Care. 2020;24(1):263.

25. Manson JJ, Crooks C, Naja M, Ledlie A, Goulden B, Liddle T, et al. COVID-19-associated hyperinflammation and escalation of patient care: a retrospective longitudinal cohort study. Lancet Rheumatol. 2020;2(10):e594-602.

26. Zhou Q, Gao Y, Wang X, Liu R, Du P, Wang X, et al. Nosocomial infections among patients with COVID-19, SARS and MERS: a rapid review and meta-analysis. Ann Transl Med. 2020;8(10):629.

27. Manohar P, Loh B, Nachimuthu R, Hua X, Welburn SC, Leptihn S. Secondary bacterial infections in patients with viral pneumonia. Front Med (Lausanne). 2020;7:420.

28. Hotchkiss RS, Monneret G, Payen D. Sepsis-induced immunosuppression: from cellular dysfunctions to immunotherapy. Nat Rev Immunol. 2013;13(12):862-74

29. Venet F, Monneret $G$. Advances in the understanding and treatment of sepsis-induced immunosuppression. Nat Rev Nephrol. 2018;14(2):121-37.

30. Ortillon M, Coudereau R, Cour M, Rimmelé T, Godignon M, Gossez M, et al. Monocyte CD169 expression in COVID-19 patients upon intensive care unit admission. Cytometry A. 2021. (in press).

31. Zhou R, To KK, Wong YC, Liu L, Zhou B, Li X, et al. Acute SARS-CoV-2 infection impairs dendritic cell and $T$ cell responses. Immunity. 2020;53(4):864-77.

32. Monneret G, Gossez M, Venet F. Sepsis and immunosenescence: closely associated in a vicious circle. Aging Clin Exp Res. 2021. (in press)

33. Rydyznski Moderbacher C, Ramirez SI, Dan JM, Grifoni A, Hastie KM, Weiskopf D, et al. Antigen-specific adaptive immunity to SARS-CoV-2 in acute COVID-19 and associations with age and disease severity. Cell. 2020;183(4):996-1012

34. Trouillet-Assant S, Viel S, Ouziel A, Boisselier L, Rebaud P, Basmaci R, et al. Type I interferon in children with viral or bacterial infections. Clin Chem. 2020;66(6):802-8.

35. Vardhana SA, Wolchok JD. The many faces of the anti-COVID immune response. J Exp Med. 2020;217(6):e20200678.

36. Liu Y, Yang Y, Zhang C, Huang F, Wang F, Yuan J, et al. Clinical and biochemical indexes from 2019-nCoV infected patients linked to viral loads and lung injury. Sci China Life Sci. 2020;63(3):364-74.

37. Liu Y, Liao W, Wan L, Xiang T, Zhang W. Correlation between relative nasopharyngeal virus RNA load and lymphocyte count disease severity in patients with COVID-19. Viral Immunol. 2020. (in press).

38. Buetti N, Wicky PH, Le Hingrat Q, Ruckly S, Mazzuchelli T, Loiodice A, et al. SARS-CoV-2 detection in the lower respiratory tract of invasively ventilated ARDS patients. Crit Care. 2020;24(1):610.

39. Wang Y, Zhang L, Sang L, Ye F, Ruan S, Zhong B, et al. Kinetics of viral load and antibody response in relation to COVID-19 severity. J Clin Invest. 2020;130(10):5235-44.

40. Huang Y, Chen S, Yang Z, Guan W, Liu D, Lin Z, et al. SARS-CoV-2 viral load in clinical samples from critically ill patients. Am J Respir Crit Care Med. 2020;201(11):1435-8.

41. Pujadas E, Chaudhry F, McBride R, Richter F, Zhao S, Wajnberg A, et al. SARS-CoV-2 viral load predicts COVID-19 mortality. Lancet Respir Med. 2020;8(9):e70

42. Xu J, Yang $X$, Yang L, Zou X, Wang Y, Wu Y, et al. Clinical course and predictors of 60-day mortality in 239 critically ill patients with COVID-19: a multicenter retrospective study from Wuhan, China. Crit Care. 2020;24(1):394.

43. Bitker L, Dhelft F, Chauvelot L, Frobert E, Folliet L, Mezidi M, et al. Protracted viral shedding and viral load are associated with ICU mortality in Covid-19 patients with acute respiratory failure. Ann Intensive Care. 2020;10(1):167.

44. Remy KE, Brakenridge SC, Francois B, Daix T, Deutschman CS, Monneret G, et al. Immunotherapies for COVID-19: lessons learned from sepsis. Lancet Respir Med. 2020;8(10):946-9.

45. Zhou F, Yu T, Du R, Fan G, Liu Y, Liu Z, et al. Clinical course and risk factors for mortality of adult inpatients with COVID-19 in Wuhan, China: a retrospective cohort study. Lancet. 2020;395(10229):1054-62.

46. Bartoletti M, Pascale R, Cricca M, Rinaldi M, Maccaro A, Bussini L, et al. Epidemiology of invasive pulmonary aspergillosis among COVID-19 intubated patients: a prospective study. Clin Infect Dis. 2020. (in press).

47. Marr KA, Platt A, Tornheim JA, Zhang SX, Datta K, Cardozo C, et al. Aspergillosis complicating severe coronavirus disease. Emerg Infect Dis. 2021;27(1):18.

48. Dupont D, Menotti J, Turc J, Miossec C, Wallet F, Richard JC, et al. Pulmonary aspergillosis in critically ill patients with Coronavirus Disease 2019 (COVID-19). Med Mycol. 2021;59(1):110-4.

49. Huang M, Wang Y, Ye J, Da H, Fang S, Chen L. Dynamic changes of T-lymphocyte subsets and the correlations with 89 patients with coronavirus disease 2019 (COVID-19). Ann Transl Med. 2020;8(18):1145.

50. Sette A, Crotty S. Adaptive immunity to SARS-CoV-2 and COVID-19. Cell. 2021;184(4):861-80.

51. Remy KE, Mazer M, Striker DA, Ellebedy AH, Walton AH, Unsinger J, et al. Severe immunosuppression and not a cytokine storm characterizes COVID-19 infections. JCI Insight. 2020;5(17):e140329.

52. Zheng M, Gao Y, Wang G, Song G, Liu S, Sun D, et al. Functional exhaustion of antiviral lymphocytes in COVID-19 patients. Cell Mol Immunol. 2020;17(5):533-5

53. Zheng HY, Zhang $M$, Yang $C X$, Zhang $N$, Wang XC, Yang XP, et al. Elevated exhaustion levels and reduced functional diversity of $T$ cells in peripheral blood may predict severe progression in COVID-19 patients. Cell Mol Immunol. 2020;17(5):541-3.

54. Leisman DE, Ronner L, Pinotti R, Taylor MD, Sinha P, Calfee CS, et al. Cytokine elevation in severe and critical COVID-19: a rapid systematic review, meta-analysis, and comparison with other inflammatory syndromes. Lancet Respir Med. 2020;8(12):1233-44.

55. Sinha P, Matthay MA, Calfee CS. Is a "cytokine storm" relevant to COVID19? JAMA Intern Med. 2020;180(9):1152-4.

56. Sinha P, Calfee CS, Cherian S, Brealey D, Cutler S, King C, et al. Prevalence of phenotypes of acute respiratory distress syndrome in critically ill patients with COVID-19: a prospective observational study. Lancet Respir Med. 2020;8(12):1209-18.

57. Blanco-Melo D, Nilsson-Payant BE, Liu WC, Uhl S, Hoagland D, Moller R, et al. Imbalanced host response to SARS-CoV-2 drives development of COVID-19. Cell. 2020;181(5):1036-45.

58. Fajnzylber J, Regan J, Coxen K, Corry H, Wong C, Rosenthal A, et al. SARS-CoV-2 viral load is associated with increased disease severity and mortality. Nat Commun. 2020;11(1):5493.

59. Veyer D, Kerneis S, Poulet G, Wack M, Robillard N, Taly V, et al. Highly sensitive quantification of plasma SARS-CoV-2 RNA shelds light on its potential clinical value. Clin Infect Dis. 2020. (in press).

60. Blot M, Jacquier M, Manoha C, Piroth L, Charles PE, Pneumochondrie study g. Alveolar SARS-CoV-2 viral load is tightly correlated with severity in COVID-19 ARDS. Clin Infect Dis. 2020. (in press).

61. Xu D, Zhou F, Sun W, Chen L, Lan L, Li H, et al. Relationship between serum SARS-CoV-2 nucleic acid(RNAemia) and organ damage in COVID19 patients: a cohort study. Clin Infect Dis. 2020. (in press).

62. Bermejo-Martin JF, Gonzalez-Rivera M, Almansa R, Micheloud D, Tedim AP, Dominguez-Gil M, et al. Viral RNA load in plasma is associated with critical illness and a dysregulated host response in COVID-19. Crit Care. 2020;24(1):691.

\section{Publisher's Note}

Springer Nature remains neutral with regard to jurisdictional claims in published maps and institutional affiliations. 